\title{
Assessment of different fitting methods for in-vivo bi-component T2* analysis of human patellar tendon in magnetic resonance imaging
}

Fang Liu

Richard Kijowski

University of Wisconsin-Madison, USA

Corresponding author:

Fang Liu

Radiology, University of Wisconsin-Madison

1111 Highland Ave

53705 Madison, USA

E-mail: fliu37@wisc.edu

\section{Summary}

Purpose: To investigate the robustness of four fitting methods for bi-component effective spin-spin T2 (T2*) relaxation time analysis of human patellar tendon.

Methods: A three-dimensional (3D) cone ultrashort echo-time (UTE) sequence was performed on the knees of ten healthy volunteers at 3.0T. Four fitting methods incorporating either Gaussian or Rician noise distribution were used for voxel-by-voxel bi-component T2* analysis of the patellar tendon. The $\mathrm{T}^{*}$ for the short relaxing $\left(T^{*}, s\right)$ and long relaxing $\left(T^{*}{ }_{2, l}\right)$ water components and the fraction of the short relaxing water component $\left(f_{s}\right)$ were measured, and different fitting methods were compared using Friedman's and Wilcoxon signed rank tests. A numerical simulation study was also performed to predict the accuracy and precision of bi-component T2* parameter estimation in tendon at different signal-tonoise ratios (SNR) levels.

Results: The average $T^{*}{ }_{2, s}, T^{*}{ }_{2, l}, f_{s}$ of human patellar tendon were $1.5 \mathrm{~ms}, 30 \mathrm{~ms}$, and $80 \%$ respectively. Incorporating different noise models and fitting methods influenced the measured bi-component T2* $^{*}$ parameters. Fitting methods incorporating Rician noise were superior to traditional fitting methods for bi-component T2* analysis especially at lower SNR. $f_{s}$ and $T^{*}{ }_{2, s}$ were less sensitive than $T^{*} 2,1$ to noise at even moderate and low SNR. The result of the in-vivo bi-component T2* analysis of tendon agreed well with numerical simulations.

Conclusion: Our study demonstrated the use of a 3D cone UTE sequence to perform in vivo voxel- by-voxel bi-component T2* analysis of human patellar tendon. Incorporating Rician noise was useful for improving bi-component T2* analysis especially at lower SNR.

Level of evidence: IV.

KEY WORDS: tendon, T2 relaxation time, bi-component, curve fitting, noise.

\section{Introduction}

Various imaging methods including radiographs, ultrasound, computed tomography, and conventional and quantitative magnetic resonance (MR) imaging can be used to evaluate musculoskeletal tissues. However, only quantitative MR imaging can be used to provide information regarding tissue composition and microstructure. Thus, these imaging techniques play an important role in the non-invasive assessment of disease-related and treatment-related changes in cartilage, bone, meniscus, and tendon. Spin-spin (T2) and effective spin-spin (T2*) relaxation times are the most commonly used quantitative MR method used in musculoskeletal imaging and have been shown to useful for evaluating tissue composition and microstructure ${ }^{1-4}$. However, changes in T2 and $\mathrm{T}^{*}$ are nonspecific and can be caused by multiple factors including hydration, macromolecular content, and tissue anisotropy with comparable changes occurring in disparate settings ${ }^{5-9}$

Bi-component T2 and T2* mapping techniques have been used to improve the specificity of T2 analysis by assessing the individual water components of musculoskeletal tissues 10-22. Bi-component T2 and T2* mapping methods have measured two distinct T2 components in cartilage assumed to represent short relaxing water bound to the macromolecular matrix and long relaxing bulk water 12-14,21,22. Bi-component T2* mapping methods have been used in cortical bone to differentiate between water bound to the organic matrix and free water in the Haversian systems 15-17, Bi-component T2 and T2* mapping methods have also been used in the meniscus to differentiate between macromolecular bound water and bulk water 18-20.

In tendon, recent studies using bi-component T2* mapping techniques have measured two distinct T2 components representing short relaxing water bound to the highly organized collagen fibers and long relax- 
ing bulk water ${ }^{19}$. Chang et al. detected short and long relaxing water components in histologically normal human cadaveric Achilles tendon with a T2* of $1.8 \mathrm{~ms}$ and $9.2 \mathrm{~ms}$ and a fraction of $79.2 \%$ and $20.8 \%$ respectively ${ }^{23}$. The Authors later reported that bi-component $\mathrm{T}^{*}$ parameters did not change with tensile loading of the Achilles tendon ${ }^{24}$. Jarus et al. compared single-component and bi-component T2* analysis of the Achilles tendon in human subjects and found that the bi-component analysis provided greater diagnostic performance for distinguishing between normal and pathologic tendon 25.

However, multiple factors may affect estimations of bi-component T2 and T2* parameters within musculoskeletal tissues. Bi-component T2 measurements have been shown to be strongly influenced by experimental details such as the field strength, gradient system, pulse sequence, imaging parameters, and specimen preparation 26,27 . Bouhrara et al. also demonstrated that accurate estimation of bi-component T2 parameters requires sufficient signal-to-noise (SNR) ratio and proper noise modeling which is particularly important when estimating pixel-by-pixel spatial variations across a tissue sample ${ }^{28}$. In their study, simulations and imaging experiments on ex vivo phantoms and bovine nasal cartilage specimens were used to compare multiple model fitting methods and to investigate the sensitivity and robustness of bicomponent T2* measurements at different SNR levels. Their conclusion resided in the importance of incorporating Rician noise models in bi-component T2* analysis of cartilage.

However, questions remain regarding the effectiveness of using Rician noise models for estimating bi-component T2* parameters in in vivo musculoskeletal imaging. Furthermore, the applicability of these models for imaging tendon which has different tissue composition and microstructure than bovine nasal cartilage requires further investigation. In this study, we investigated the applicability of translating observations derived from simulations and ex vivo experiments into in-vivo tendon imaging to provide insight into the best fitting methods for use in bi-component T2* analysis. More specifically, our study compared different curve fitting methods incorporating different noise models for measuring bicomponent $\mathrm{T}^{*}$ parameters of the patellar tendon in a numerical simulation study and in healthy volunteers using a three-dimensional (3D) cone ultra-short echotime (UTE) sequence.

\section{Methods}

\section{Bi-Component signal model and fitting methods}

A bi-component exponential signal model was used to characterize the short (denoted as s) and long (denoted as I) relaxing water components in patellar tendon, given by

$$
\begin{gathered}
S(T E)=S_{N}(T E)+\varepsilon(T E) \\
S_{N}(T E)=A\left(f_{s} \times e^{-T E / T_{2, s}^{*}}+\left(1-f_{s}\right) \times e^{-T E / T_{2, l}^{*}}\right)
\end{gathered}
$$

where $S$ is the acquired signal at varying TEs with noise, $S_{N}$ is the underlying noise free signal, $A$ is the combined factor of proton density and hardware gain, $T^{*}{ }_{2, s}$, and $T^{*}{ }_{2, l}$ are $\mathrm{T} 2{ }^{*}$ for the short and long relaxing water components respectively, and $f_{s}$ is the water fraction for the short water component, defined as the ratio of the short water content to the total water (short + long) content. $\varepsilon(T E)$ is the noise term at the individual echo with noise between each echo assumed to be independent.

Four different fitting methods using the non-linear least square minimization of the acquired signal and theoretical model were compared. Under the simple assumption of Gaussian distributed noise of the image signal, the first method, $M 1$, is the traditional approach where $\left|S-S_{N}\right|$ is minimized. Under the assumption of a Gaussian distributed noise with equal variance in both real and imaginary channel of the acquired signal, the noise of the magnitude image can be described using Rician distribution as

$$
P\left(S \mid S_{N}, \sigma\right)=\frac{S}{\sigma^{2}} e^{\frac{-\left(S_{N}^{2}+S^{2}\right)}{2 \sigma^{2}}} I_{0}\left(\frac{S_{N} \cdot S}{\sigma^{2}}\right)
$$

where $\sigma$ is the noise standard deviation. $I_{0}$ denotes the modified zero order Bessel function of the first kind. The second fitting method, $\mathrm{M} 2$, is minimization of $\sqrt{\left|S^{2}-S_{N}^{2}-2 \sigma^{2}\right|}$ based on McGibney's scheme for corjartsson et al. ${ }^{30}$ also introduced a simple correction scheme, M3, for minimization of $\left|\sqrt{S^{2}-\sigma^{2}}-S_{N}\right|$. Bouhrara et.al. ${ }^{28}$ recently introduced a fitting scheme, M4, with the minimization of $\left|S-S_{R}\right|$ for

$$
S_{R}=\int_{0}^{\infty} S \cdot P\left(S \mid S_{N}, \sigma\right) d S=\sigma \sqrt{\frac{\pi}{2}} e^{-\alpha}\left((1+2 \alpha) I_{0}(\alpha)+2 \alpha I_{1}(\alpha)\right)
$$

where $\alpha=\left(\frac{S_{N}}{2 \sigma}\right)^{2}$ and $I_{1}$ is the modified first order Bessel function of the first kind. In all the fitting methods, the image SNR was estimated for the first echo signal and defined as 28,29

$$
S N R=\frac{\sqrt{S_{N}^{2}-2 \sigma^{2}}}{\sigma}
$$

where the noise standard deviation $\sigma$ was determined as the mean signal of background regions of all images acquired at each TE divided by $\sqrt{\pi / 2} 31$.

\section{Simulation study}

Numerical simulations were performed to compare accuracy and precision of bi-component T2* parameter estimation using four different fitting methods. Theoretical multi-echo spin-echo signal data were generated based on bi-exponential decay equations as described in Eq.(1) for the real part of the signal. To simulate the Rician noise, the imaginary part of signal was created using only the zero mean Gaussian noise with the same noise standard deviation as the real part, and the magnitude of the complex signal was used as the final theoretical signal ${ }^{28}$. The fi- 
nal signal was fitted with the four different fitting methods and corresponding bi-component T2* parameters were estimated for each individual method. A total of 10,000 instances of noise were added to the signal in Monte-Carlo simulations to measure the accuracy and precision of the parameter estimation for $f_{s}, T^{*}{ }_{2, s}$, and $T_{2, l}^{*}$. The performance measure included the percentage error, which was defined as the difference between the estimated mean and true parameter value divided by the true parameter value. The same simulation was repeated with different levels of added noise at SNR $=20,50$ and 90 .

The set of model parameters used in the numerical simulations was chosen to mimic bi-component T2* parameters of tendon previously published in the literature $^{24}$. Accordingly, parameters of the two water pools were chosen as follows: $f_{s}=80 \%, T^{*}{ }_{2, s}=1 \mathrm{~ms}$, and $T^{*}{ }_{2, l}=20 \mathrm{~ms}$. The signals were generated using the experimental design of the in vivo protocol described in the next subsection.

\section{In vivo study}

The in-vivo study was performed in compliance with Health Insurance Portability and Accountability Act (HIPPA) regulations and with approval from the University Institutional Review Board. The study met the ethical standards of the Muscle, Tendon, and Ligament Journal ${ }^{32}$. All subjects signed written informed consent prior to their participation in the study. The study group consisted of 10 healthy volunteers (6 males with an average age of 28 years and 4 females with an average age of 27 years) who had no history of prior knee pain, trauma, or surgery.

All subjects underwent an MR examination of the right knee on the same 3.0T scanner (Discovery MR750, GE Healthcare, Waukesha, WI) using an 8channel phased-array extremity coil (InVivo, Orlando, FL). Foam padding was used to firmly secure the knee within the coil to minimize subject motion during the MR examination. The patellar tendon was imaged in the sagittal plane using a 3D gradient-echo-based multi-echo UTE sequence ${ }^{33}$. The UTE sequence utilized a k-space sampling scheme of a center-out twisted $3 D$ cone trajectory which allowed a minimal echo time (TE) of $0.03 \mathrm{~ms}$. A total of 16 echoes were acquired at TEs of $0.03,0.1,0.8,1.6,4.3,6.0,8.0$, $10.0,14.0,16.0,18.0,20.0,24.0,26.0,28.0,30.0 \mathrm{~ms}$ based upon TE selection from a previous tendon study 24 . For each acquisition, the same repetition time (TR) was used, and four echoes were acquired. Other imaging parameters included $40 \mathrm{~ms}$ TR, $20^{\circ}$ flip angle, $16 \mathrm{~cm}$ field-of-view, $\pm 150 \mathrm{KHz}$ readout bandwidth, $1.21 \mathrm{~ms}$ readout length, 256x256 in-plane matrix, $3 \mathrm{~mm}$ slice thickness, one excitation, and 10 slices covering the entire patellar tendon. The total imaging time was approximately 12 minutes.

All images were registered to the first echo using a rigid registration method implemented in Elastix software to correct for subject motion between scans ${ }^{34}$. The patellar tendon of all 10 subjects on all sagittal images was manually segmented by an experienced research assistant under the supervision of a muscu- loskeletal radiologist using an in-house software program developed in Matlab (Matlab 2010b, MathWorks Inc, Natick, MA). A voxel-by-voxel signal curve fitting was performed to obtain 3D parameter maps for $f_{s}$, $T^{*}{ }_{2, s}$, and $T^{*}{ }_{2, l}$. The tendon masks were then superimposed over the bi-component $\mathrm{T}^{*}$ maps to measure the mean and standard deviation of $f_{s}, T^{*}{ }_{2, s}$, and $T^{*}{ }_{2, l}$ of the entire patellar tendon for all subjects.

The voxel-by-voxel curve fitting was performed using an in-house $T 2 *$, mapping software program developed based on the non-linear least square "fmincon" function implemented in Matlab. The initial parameter guess $T_{2, s}^{*}=1 \mathrm{~ms}, T_{2,1}^{*}=20 \mathrm{~ms}$ and $f_{s}=80 \%$ based on previous published tendon studies was used in the iterative optimization ${ }^{24}$. The parameter searching space were [0ms, 20ms], [0ms, $100 \mathrm{~ms}$ ] and [0\%, $100 \%]$ for $T_{2, s}^{*}$, and $T^{*}{ }_{2, l}$, and $f_{s}$ respectively, reflecting a reasonable parameter range within the patellar tendon. The algorithm converging iterations were continued until the step size between the successive estimates was small $\left(<10^{-8}\right)$, the object function was small $\left(<10^{-8}\right)$, or total iteration of 1000 was achieved. The voxels failing the converging iteration criteria or converged at the parameter searching space boundaries were recorded as the outliers, and the corresponding bi-component $\mathrm{T}^{*}$ parametric values were substituted with the value of 0 or boundary values respectively. The outliers were excluded prior to the statistical analysis to avoid statistical bias, and the percentage of outliers was recorded for each subject. To demonstrate the quality of the curve fitting models for the acquired image data, quality of fit measures were compared for the mean signal from a homogenous ROI chosen on a sagittal slice in the central portion of the patellar tendon to avoid partial volume effect with adjacent tissues. The relative residual error defined as the percentage of the residual error to the signal at different echo times was used to measure the fitting quality of the models at individual echoes. The overall measure of goodness-of-fit was tested for all the four methods by using chi-square, $\chi^{2}$, value. The SNR was calculated on all subjects and referred to as $S_{N R}$ normal. To estimate the model fitting robustness at different SNR levels, a set of repeat scans were performed on one subject using the same imaging parameters except for a $1.0 \mathrm{~mm}$ slice thickness to decrease image SNR (referred to as SNR low $_{\text {). Anoth- }}$ er set of repeat scans were performed on the same subject using the same imaging parameters except for four excitations to increase image SNR (referred to as SNR high $_{\text {). }}$

\section{Statistical analysis}

Statistical analysis was performed using Matlab. Friedman's tests were used to compare the means and standard deviations of $f_{s}, T^{*}{ }_{2, s}$, and $T^{*}{ }_{2, l}$ of the entire patellar tendon of all subjects for the four fitting methods. The Friedman's test is a non-parametric one-way ANOVA test used to compare more than two groups. For those bi-component $\mathrm{T}^{*}$ parameters in which there was a significant difference between the four models, Wilcoxon signed rank tests were used 
for pairwise comparison to determine if there was significant differences between any two of the four fitting methods. The Holm-Bonferroni correction method was used to adjust all $p$-values to account for comparison of multiple MR parameters within the knee joint ${ }^{35}$.

\section{Results}

Figure 1 demonstrates the accuracy and precision of bi-component $\mathrm{T}^{*}$ parameter estimation when using four different fitting methods at different SNR levels in Monte-Carlo simulation. At low SNR (top row, SNR=20), M1, M3 and M4 provided more accurate estimation for $f_{s}(1.6 \%, 1.3 \%$ and $0.1 \%$ error $)$ and $(0.9 \%, 0.1 \%$ and $-2.2 \%$ error) compared to that of M2. M2 provided biased estimation for $f_{s}$ (5.4\% error) and $T_{2, s}^{*}(4.3 \%$ error $)$ and more estimation uncertainty (i.e. less precision) for both parameters indicated by a much broader spectrum. M3 $(11.8 \%$ error) and M4 (-0.1\% error) provided better estimation accuracy of $T^{*}{ }_{2, l}, \mathrm{M} 3$ than $\mathrm{M} 1$ (44.6\% error) and M2 $(21.4 \%$ error) at low SNR. In addition, the peak at $100 \mathrm{~ms}$ in the estimation of $T^{*}{ }_{2, l}$ indicates that all of the four fitting methods yield small amount of estimation outliers at low SNR. At moderate SNR (middle row, SNR=50), all four fitting methods provided accurate estimation for $f_{s}(0.4 \%, 0.6 \%, 0.1 \%$ and $0.1 \%$ error $), T_{2, s}^{*}(0.5 \%$, $0.4 \%, 0.1 \%$ and $0.1 \%$ error $)$, and $T^{*}{ }_{2, l}(6.2 \%,-0.3 \%$,
$0.4 \%$ and $0.6 \%$ error), while M2 provided slightly larger estimation uncertainty. At high SNR (bottom row, SNR=90), all four fitting methods provided accurate estimation for $f_{s}(0.1 \%, 0.2 \%, 0 \%$ and $0 \%$ error $), T^{*}{ }_{2, s}$ $(0.1 \%, 0.1 \%,-0.1 \%$ and $0 \%$ error $)$ and $T_{2, l}^{*}(1.7 \%$, $0.5 \%, 0 \%$ and $0 \%$ error), while $\mathrm{M} 2$ provided slightly larger estimation uncertainty. Overall, the accuracy and precision of bi-component $\mathrm{T}^{*}$ parameter estimation increased with increasing level of SNR for all four fitting methods.

Figure 2 shows sagittal images at all 16 echoes acquired using the 3D cone UTE sequence. The mean SNR of the patellar tendon for all subjects was 54.5 with a standard deviation of 7.6 and a range between 41.3 and 70.4. There was a monotonic decay of the MR signal of the patellar tendon with much stronger decay occurring prior to $4.3 \mathrm{~ms}$ than after $4.3 \mathrm{~ms}$. Other tissues including cartilage, bone marrow fat, and subcutaneous fat also demonstrated signal decay from the short echoes to the long echoes.

Figure $3 \mathrm{~A}$ shows the mean signal from a ROI in the central portion of the patellar tendon. All four fitting methods showed visually good fit. However, the overall goodness-of-fit was better (i.e. lower $\chi^{2}$ value) for $\mathrm{M} 1\left(\chi^{2}=160\right), \mathrm{M} 3\left(\chi^{2}=140\right)$ and $\mathrm{M} 4\left(\chi^{2}=145\right)$ than that of $\mathrm{M} 2\left(\chi^{2}=389\right)$. Figure 3B demonstrates the relative residual errors in percentage for the four fitting methods. For all fitting methods, there was unbiased signal fitting prior to $5 \mathrm{~ms}$ with no more than $5 \%$ residual error, while the fitting had relatively larger error and

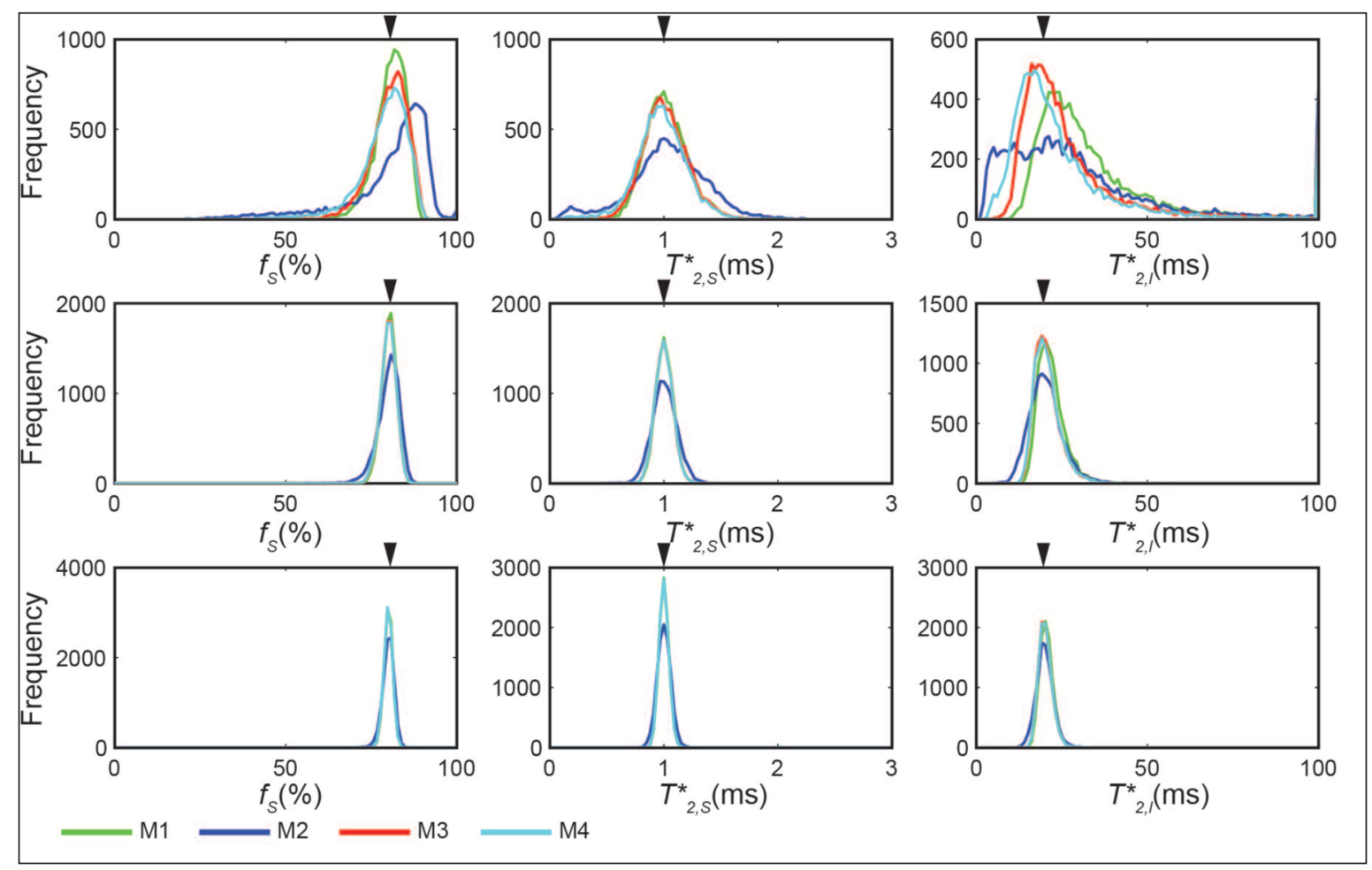

Figure 1. Histogram of bi-component T2* parameter estimation in Monte-Carlo simulation at different SNR levels (Top row: SNR=20, Middle row: $S N R=50$, Bottom row: $S N R=90$ ). The dark arrows indicate the ground truth value for each individual parameter. 


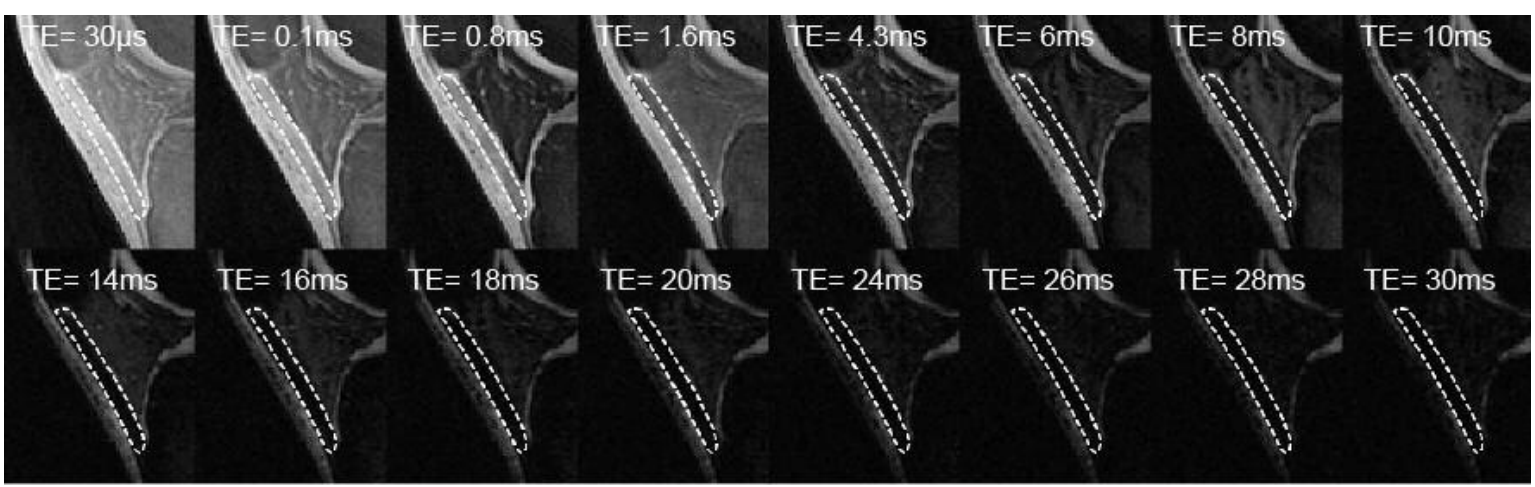

Figure 2. Sagittal UTE images from the central portion of the patellar tendon of a 31-years-old healthy male volunteer. The white dash line delineates the region of patellar tendon after manual segmentation. The series of images demonstrate signal decay within the patellar tendon at different TEs from the shortest echo $(T E=30 \mu \mathrm{s})$ to the longest echo (TE=30ms).

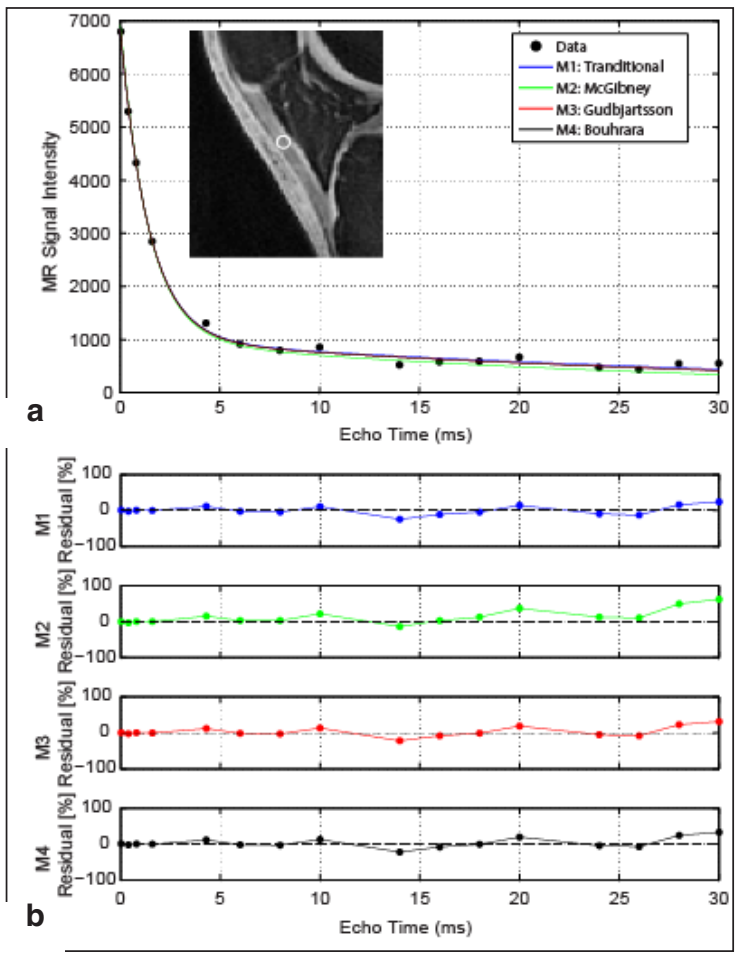

Figure 3. (a) The average signal from an ROI (white circle) in the patellar tendon shows a monotonically signal decay from $30 \mu \mathrm{s}$ to $30 \mathrm{~ms}$. Rapid signal decay is shown at TEs prior to $5 \mathrm{~ms}$ followed by slow signal decay. All four model fitting methods show good fit to the data. (b) Fitting methods M1, M3 and M4 show almost identical fit quality with small relative residual errors. Fitting method M2 shows similar fit quality as the other three methods at TEs prior to $5 \mathrm{~ms}$, but slightly larger residual errors at later echoes.

more uncertainty after $5 \mathrm{~ms}$. M1, M3 and M4 showed visually almost identical fitting quality with small relative residual errors at all echoes. M2 showed similar fitting quality as the other three methods prior to $5 \mathrm{~ms}$ but slightly larger residual errors at the later echoes. Table I shows the mean and percentage of outliers of $f_{s}, T^{*}{ }_{2, s}$, and $T^{*}, l$ of the patellar tendon of all subjects for all four fitting methods. M2 had the largest (13.53\%) and $\mathrm{M} 1$ the smallest $(6.05 \%)$ mean percentage of outliers. There was a significant difference between the four fitting methods for the mean $f_{s}(p=0.001), T^{*}{ }_{2, s}(p$ $=0.04)$, and $T_{2, l}^{*}(p=0.004)$ of the patellar tendon. M1 provided significantly higher mean $f_{s}(p<0.05)$ and $T^{*}{ }_{2, l}$ $(p<0.05)$ than $\mathrm{M} 3$ and M4. M3 provided significantly higher mean $f_{s}(p<0.05)$ and $T^{*}{ }_{2, l}(p<0.05)$ than M4, while M2 provided significantly higher of $T_{2, s}^{*} \quad(p<0.05)$ than M1, M3 and M4.

Table I also shows the standard deviations of $f_{s}, T^{*}{ }_{2, s}$, and $T_{2, l}^{*}$ of the patellar tendon of all subjects for all four fitting methods. There was a significant difference between the four fitting methods for the standard deviation of $T^{*}{ }_{2, s}(p<0.001), T^{*}{ }_{2, l}(p<0.0001)$, and $f_{s}(p<$ $0.001)$ of the patellar tendon. M2 provided significantly higher standard deviation of $f_{s}(p<0.05), T^{*}{ }_{2, s} \quad(p<0.05)$, and $T^{*}{ }_{2, l}(p<0.05)$ than M1, M3 and M4. M1 provided significantly higher standard deviation of $T_{2, l}^{*}(p<0.05)$ than M3 and M4.

The SNR of the patellar tendon was 84.2, 48.3, and 21.3 for the $S N R_{\text {high }}, S_{N R}$ normal, and $S_{N R}$ low images respectively. Figure 4, 5, and 6 show $f_{s}, T^{*}{ }_{2, s}$, and $T^{*}{ }_{2, l}$ maps of the central portion of the patellar tendon in one subject at different SNR levels. Tables 2 and 3 show the mean and standard deviations of $f_{s}, T^{*}{ }_{2, s}$ and $T^{*}{ }_{2, l}$ of all patellar tendon voxels in one subject at different SNR levels. There was a dramatic increase of mean $T^{*}{ }_{2, l}$ with decrease in image SNR, while $f_{s}$ and $T^{*}{ }_{2, s}$ remained relatively the same. There was an increase in the standard deviation of $f_{s}, T^{*}{ }_{2, s}$, and $T^{*}{ }_{2, l}$ with decrease in image SNR. The standard deviation of $f_{s}$, $T^{*}{ }_{2, s}$, and $T^{*}{ }_{2, l}$ was much larger for M2 than for M1, M3 and M4.

\section{Discussion}

Various imaging methods including ultrasound and conventional MR imaging have been used to evaluate tendon ${ }^{36}$. However, tendon and other musculoskeletal tissues containing abundant highly organized col- 


\section{F. Liu et al.}

Table I. Mean and standard deviation and mean percentage of outliers of $f_{s}, T_{2, s}^{*}$, and $T_{2, l}^{*}$ of the patellar tendon of all 10 subjects for all four fitting methods.

\begin{tabular}{ll|ll|ll|ll}
\hline & \multicolumn{3}{l}{ Outliers [\%] } & \multicolumn{3}{c}{$f_{s}[\%]$} & \multicolumn{2}{c}{$T_{2, s}^{*_{2}[\mathrm{~ms}]}$} & $T_{2, I}^{*_{2}[\mathrm{~ms}]}$ \\
\hline & mean & mean & SD & mean & SD & mean & SD \\
\hline M1 & 6.05 & 82.45 & 10.08 & 1.54 & 0.56 & 31.21 & 22.81 \\
M2 & 13.53 & 82.49 & 11.35 & 1.68 & 0.69 & 29.96 & 26.15 \\
M3 & 9.17 & 81.88 & 10.14 & 1.54 & 0.56 & 28.42 & 18.13 \\
M4 & 9.31 & 81.72 & 10.17 & 1.54 & 0.56 & 28.11 & 18.10 \\
\hline
\end{tabular}
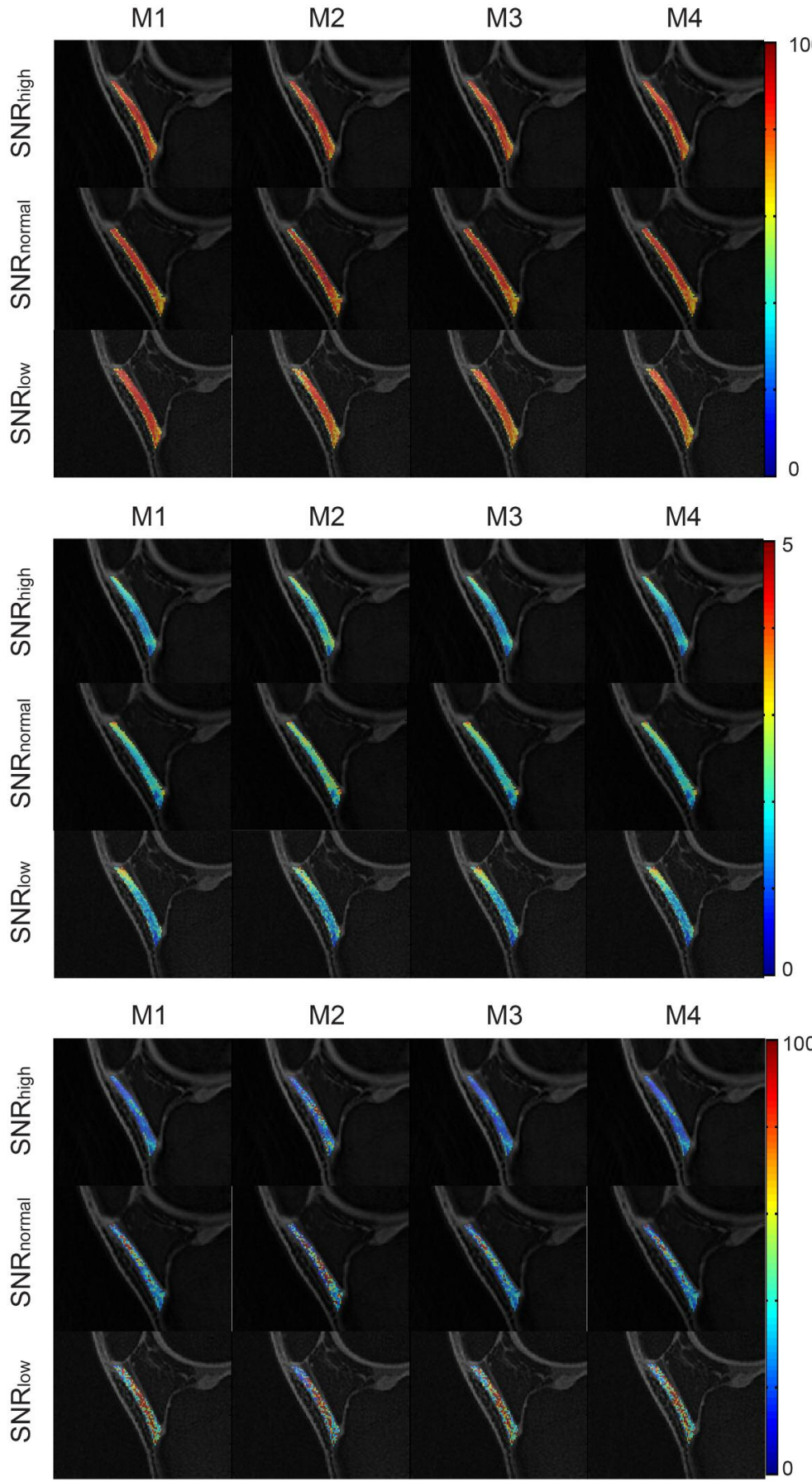

100 Figure 4. Sagittal $f_{s}(\%)$ maps of the central portion of the patellar tendon in a 29year-old healthy female volunteer show the noise performance at three different SNR levels for the four different fitting methods.

Figure 5. A sagittal $\boldsymbol{T}^{*_{2, l}}$ (ms) maps of the central portion of the patellar tendon in a 29-year-old healthy female volunteer show the noise performance at three different SNR levels for the four different fitting methods.

Figure 6. A sagittal $\boldsymbol{T}^{*}{ }_{2, l}$ (ms) maps of the central portion of the patellar tendon in a 29-year-old healthy female volunteer show the noise performance at three different SNR levels for the four different fitting methods. 
Assessment of different fitting methods for in vivo bi-component $T 2 *$ analysis of human patellar tendon in magnetic resonance imaging

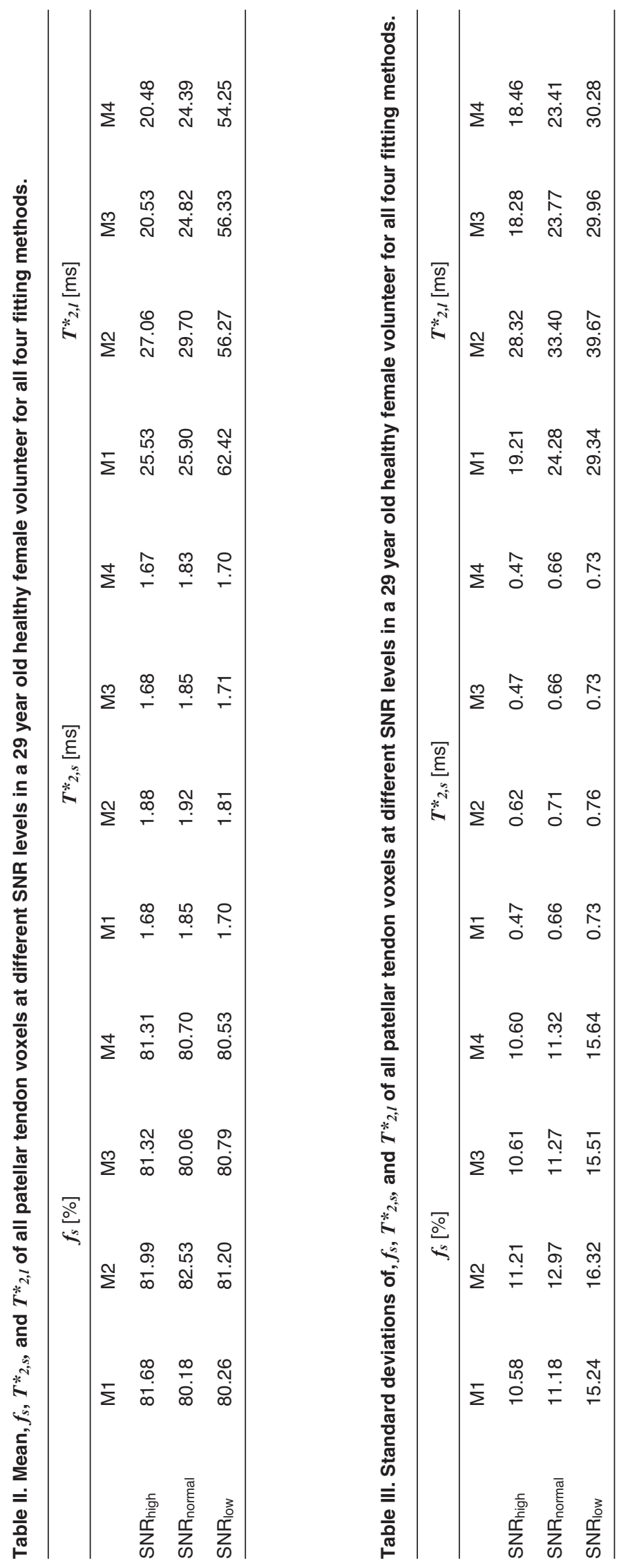


lagen fibers are difficult to assess using quantitative MR techniques due to their extremely rapid signal decay. New MR techniques including ultra-short echotime (UTE) imaging 10,37,38, variable echo time (VTE) imaging 25,39 and zero echo-time (ZTE) imaging 40,41 have been recently developed to capture the rapidly decaying signal within musculoskeletal tissues. The UTE sequence used in our study utilized a center-out twisted 3D cone k-space trajectory which has been shown to increase sampling uniformity, decrease undersampling artifact, and provide better SNR performance when compared to traditional $2 D$ and $3 D$ radial UTE sequences ${ }^{33}$. The sequence has had successful applications in sodium imaging 42,43 and ex-vivo Achilles tendon imaging ${ }^{24}$. In our study, the 3D cone UTE sequence provided complete anatomic coverage of the patellar tendon in human subjects at 3.0T with clinically feasible spatial resolutions and scan times and with an SNR of 54.5 , which has been shown to be adequate to accurately estimate bi-component T2* parameters from previous literature ${ }^{28}$ and the results of our simulation study.

Recent studies have demonstrated that bi-component exponential signal models are better suited than single-component models for evaluating tendon ${ }^{19,23-25}$. Bi-component models have detected two distinct T2 components within tendon representing fast relaxing water bound to the highly organized collagen fibers and slow relaxing bulk water. The $\mathrm{T}^{*}$ of the two water components in tendon have varied in different studies between $0.3 \mathrm{~ms}$ and $1.3 \mathrm{~ms}$ for the short component and $8.2 \mathrm{~ms}$ and $20.4 \mathrm{~ms}$ for the long component 19,23-25. The differences in measured T2* parameters is likely due to multiple factors including different field strengths, vender platforms, sequence designs, imaging parameters, imaging conditions, and tendon types. Our study reported a short T2* of around $1.5 \mathrm{~ms}$ and a long $\mathrm{T} 2^{\star}$ of around $28 \mathrm{~ms}$ which is close to the values of $0.9 \mathrm{~ms}$ and $20.4 \mathrm{~ms}$ reported by Chang et al. ${ }^{24}$. This may be due to the fact that both studies used similar 3D Cone UTE sequences although the sequence used by Chang et al. to evaluate ex-vivo Achilles tendon in human cadavers had a scan time of 60 minutes which was much longer than the 12 minute scan time of our sequence.

Bi-component $\mathrm{T}^{*}$ analysis has been shown to provide better diagnostic performance than single-component $\mathrm{T}^{*}$ analysis for distinguishing between patients with Achilles tendinopathy and asymptomatic volunteers ${ }^{44}$. Furthermore, previous studies have found a significant increase in short $\mathrm{T}^{*}$ in pathologic tendon when compared to normal tendon, which is specifically due to disruption of the highly organized collagen fiber network, but no significant difference in long T2* 25,39. Accurate estimations of bi-component T2* parameters requires sufficient SNR and proper noise modeling which is particularly important when estimating pixel-by-pixel spatial variations across a tissue sample ${ }^{28}$. Both our simulation and in-vivo studies showed that $T^{*}{ }_{2, l}$ in tendon has much greater spatial variation, as indicated by the standard deviation of image voxels, and much higher sensitivity to noise when compared to $T^{*}{ }_{2, s}$ and $f_{s}$. Thus, the diagnostic performance of $T^{*}, l$, for detecting tendon pathology could be substantially reduced by both low image SNR and by large spatial variations (i.e. uncertainty) in the setting of normal or high image SNR ${ }^{21}$. One explanation for the large uncertainty and high sensitivity to noise of $T_{2, l}^{*}$ is the relatively small fraction of the long $\mathrm{T}^{*}$ component in tendon which provides little MR signal at later echoes. More accurate estimations of $T^{*}, l$ may require a larger number of later acquired echoes, use of multiple excitations, or further optimization of echo number and echo spacing. However, our results in both simulation and in-vivo study also showed that $f_{s}$ and $T^{*}{ }_{2, s}$ measurements are relatively insensitive to image SNR which suggests that they may serve as robust MR parameters for evaluating tendon composition and microstructure. Our study has demonstrated that bi-component T2* measurements are influenced by the method used for curve fitting. Previous studies performed by Karlsen et al. ${ }^{45}$ and Raya et al. ${ }^{46}$ have described methods for single-component T2 analysis at low image SNR with incorporation of the Rician noise distribution. Recently, a simulation study performed by Bouhrara et al. extended the work to bi-component $\mathrm{T}^{*}$ analysis where comparison of noise performance among different fitting methods was conducted using MonteCarlo and Cramer-Rao lower bound (CRLB) simulations $^{28}$. Their results showed that the $\mathrm{M} 1$ and $\mathrm{M} 2$ fitting methods provided higher estimation bias and greater estimation uncertainty than the M3 and M4 fitting methods. Our numerical simulation and in-vivo study evaluating the patellar tendon in human subjects showed similar findings with the M2 fitting method having the greatest uncertainty which could be attributed to the fact that M2 yields neither the Rician nor Gaussian noise distribution at low SNR 30,45 . The M3 and M4 fitting methods in our study provided nearly identical performance at all SNR conditions which agrees with the simulation results from Bouhrara et al. ${ }^{28}$ and Karlsen et al. ${ }^{45}$. Thus, proper modeling of image noise may improve the accuracy of bi-component $\mathrm{T}^{*}$ measurements, especially for the long $\mathrm{T}^{*}$ component which is most severely affected by image noise. Since the M3 and M4 fitting methods are almost equally effective and M3 is less computational expensive, our general recommendation is that the M3 fitting methods should be used for in-vivo bi-component $\mathrm{T}^{*}$ analysis of tendon in human subjects.

Our study has several limitations. First, our study focused only on the patellar tendon and included only a small number of healthy volunteers. In addition, statistical analysis could not be performed to more thoroughly evaluate the influence of image SNR on bicomponent T2* measurements. Although we attempted to acquire $S N R_{\text {high }}$ scans on multiple subjects to perform statistical analysis, only one individual was able to tolerate the more than 45 minute four excitation 3D Cones UTE sequence without significant motion artifact. In addition, our study did not use phantoms to investigate bias of the different fitting methods relative to true parameter values or clinical pa- 
Assessment of different fitting methods for in vivo bi-component $T 2 *$ analysis of human patellar tendon in magnetic resonance imaging

tients with patellar tendinopathy to investigate whether the choice of fitting method and image SNR influences the diagnostic performance for distinguishing between normal and pathologic tendon.

In conclusion, our study has demonstrated the use of a 3D cone UTE sequence to perform in-vivo voxel-byvoxel bi-component $\mathrm{T}^{*}$ analysis of the patellar tendon in human subjects at 3.0T with clinically feasible spatial resolutions and scan times. Our simulation and in vivo results have shown that incorporating $\mathrm{Ri}-$ cian noise is helpful for improving bi-component T2* analysis of tendon especially at lower SNR. However, additional studies in patients with patellar tendinopathy and other tendon disorders are needed to determine whether bi-component $\mathrm{T}^{*}$ analysis can be used to detect tendon pathology and investigate the influence of the fitting method and image SNR on the identification and quantification of tendon disease.

\section{Conflict of interest}

The Author have no financial or personal relationships with other people or organizations that could inappropriately influence their work.

\section{References}

1. Dunn TC, Lu Y, Jin H, Ries MD, Majumdar S. T2 relaxation time of cartilage at MR imaging: comparison with severity of knee osteoarthritis. Radiology. 2004;232(2):592-598.

2. Patten C, Meyer RA, Fleckenstein JL. T2 mapping of muscle. Semin Musculoskelet Radiol. 2003;7(4):297-305.

3. Baum T, Joseph GB, Karampinos DC, Jungmann PM, Link TM, Bauer JS. Cartilage and meniscal T2 relaxation time as non-invasive biomarker for knee osteoarthritis and cartilage repair procedures. Osteoarthritis Cartilage. 2013;21(10):14741484.

4. Du J, Hermida JC, Diaz E, et al. Assessment of cortical bone with clinical and ultrashort echo time sequences. Magn Reson Med. 2013;70(3):697-704

5. Liess C, Lusse S, Karger N, Heller M, Gluer CC. Detection of changes in cartilage water content using MRI T2-mapping in vivo. Osteoarthritis and cartilage/OARS, Osteoarthritis Research Society. 2002;10(12):907-913

6. Watrin-Pinzano A, Ruaud JP, Olivier P, et al. Effect of proteoglycan depletion on T2 mapping in rat patellar cartilage. Radiology. 2005;234(1):162-170.

7. Nishioka H, Hirose J, Nakamura E, et al. T1rho and T2 mapping reveal the in vivo extracellular matrix of articular cartilage. Journal of magnetic resonance imaging: JMRI. 2012;35(1): 147-155.

8. Mosher TJ, Smith H, Dardzinski BJ, Schmithorst VJ, Smith MB. MR imaging and T2 mapping of femoral cartilage: in vivo determination of the magic angle effect. AJR American journal of roentgenology. 2001;177(3):665-669.

9. Goodwin DW, Wadghiri YZ, Zhu H, Vinton CJ, Smith ED, Dunn JF. Macroscopic structure of articular cartilage of the tibial plateau: influence of a characteristic matrix architecture on MRI appearance. AJR American journal of roentgenology. 2004;182(2):311-318.

10. Du J, Diaz E, Carl M, Bae W, Chung CB, Bydder GM. Ultrashort echo time imaging with bicomponent analysis. Magnetic resonance in medicine: official journal of the Society of Mag- netic Resonance in Medicine / Society of Magnetic Resonance in Medicine. 2012;67(3):645-649.

11. Reiter DA, Lin PC, Fishbein KW, Spencer RG. Multicomponent T2 relaxation analysis in cartilage. Magnetic resonance in medicine: official journal of the Society of Magnetic Resonance in Medicine/Society of Magnetic Resonance in Medicine. 2009;61(4):803-809.

12. Reiter DA, Roque RA, Lin PC, Doty SB, Pleshko N, Spencer RG. Improved specificity of cartilage matrix evaluation using multiexponential transverse relaxation analysis applied to pathomimetically degraded cartilage. NMR in biomedicine. 2011;24(10):1286-1294.

13. Liu F, Choi KW, Samsonov A, et al. Articular Cartilage of the Human Knee Joint: In Vivo Multicomponent T2 Analysis at 3.0 T. Radiology. 2015:142201.

14. Liu F, Chaudhary R, Hurley SA, et al. Rapid multicomponent T2 analysis of the articular cartilage of the human knee joint at 3.0T. J Magn Reson Imaging. 2014;39(5):1191-1197.

15. Biswas R, Bae W, Diaz E, et al. Ultrashort echo time (UTE) imaging with bi-component analysis: bound and free water evaluation of bovine cortical bone subject to sequential drying Bone. 2012;50(3):749-755.

16. Bae WC, Chen PC, Chung CB, Masuda K, D'Lima D, Du J. Quantitative ultrashort echo time (UTE) MRI of human cortical bone: correlation with porosity and biomechanical properties. Journal of bone and mineral research: the official journal of the American Society for Bone and Mineral Research. 2012;27 (4):848-857.

17. Li S, Chang EY, Bae WC, et al. Ultrashort echo time bi-component analysis of cortical bone-a field dependence study. Magn Reson Med. 2014;71(3):1075-1081.

18. Juras V, Apprich S, Zbýň S, et al. Quantitative MRI analysis of menisci using biexponential T2 * fitting with a variable echo time sequence. Magn Reson Med. 2013.

19. Diaz E, Chung CB, Bae WC, et al. Ultrashort echo time spectroscopic imaging (UTESI): an efficient method for quantifying bound and free water. NMR Biomed. 2012;25(1):161-168.

20. Liu F, Samsonov A, Wilson JJ, Blankenbaker DG, Block WF, Kijowski R. Rapid in vivo multicomponent T2 mapping of human knee menisci. J Magn Reson Imaging. 2015.

21. Williams A, Qian $Y$, Bear $D, C h u C R$. Assessing degeneration of human articular cartilage with ultra-short echo time (UTE) $\mathrm{T}^{*}$ mapping. Osteoarthritis and cartilage/OARS, Osteoarthritis Research Society. 2010;18(4):539-546.

22. Shao $\mathrm{H}$, Chang $\mathrm{EY}$, Pauli $\mathrm{C}$, et al. UTE bi-component analysis of $\mathrm{T}^{*}$ relaxation in articular cartilage. Osteoarthritis and cartilage/OARS, Osteoarthritis Research Society. 2016;24(2):364373.

23. Chang EY, Du J, Statum S, Pauli C, Chung CB. Quantitative bi-component $\mathrm{T2}^{*}$ analysis of histologically normal Achilles tendons. Muscles Ligaments Tendons J. 2015;5(2):58-62.

24. Chang EY, Du J, Iwasaki K, et al. Single- and Bi-component $\mathrm{T}^{*}$ analysis of tendon before and during tensile loading, using UTE sequences. J Magn Reson Imaging. 2015;42(1):114120.

25. Juras V, Apprich S, Szomolanyi P, Bieri O, Deligianni X, Trattnig S. Bi-exponential T2 analysis of healthy and diseased Achilles tendons: an in vivo preliminary magnetic resonance study and correlation with clinical score. European radiology. 2013:23(10):2814-2822.

26. Zheng S, Xia Y. On the measurement of multi-component T2 relaxation in cartilage by MR spectroscopy and imaging. Magnetic resonance imaging. 2010;28(4):537-545.

27. Wang N, Xia Y. Dependencies of multi-component T2 and T1p relaxation on the anisotropy of collagen fibrils in bovine nasal cartilage. J Magn Reson. 2011;212(1):124-132.

28. Bouhrara M, Reiter DA, Celik $\mathrm{H}$, et al. Incorporation of rician noise in the analysis of biexponential transverse relaxation in 
cartilage using a multiple gradient echo sequence at 3 and 7 tesla. Magn Reson Med. 2015;73(1):352-366.

29. McGibney G, Smith MR. An unbiased signal-to-noise ratio measure for magnetic resonance images. Medical physics. 1993;20(4):1077-1078.

30. Gudbjartsson H, Patz S. The Rician distribution of noisy MRI data. Magn Reson Med. 1995;34(6):910-914.

31. Sijbers J, den Dekker AJ. Maximum likelihood estimation of signal amplitude and noise variance from MR data. Magnetic resonance in medicine: official journal of the Society of Magnetic Resonance in Medicine / Society of Magnetic Resonance in Medicine. 2004;51(3):586-594.

32. Padulo J, Oliva F, Frizziero A, Maffulli N. Muscles, Ligaments and Tendons Journal - Basic principles and recommendations in clinical and field Science Research: 2016 Update. MLTJ. 2016;6(1):1-5.

33. Gurney PT, Hargreaves BA, Nishimura DG. Design and analysis of a practical 3D cones trajectory. Magn Reson Med. 2006;55(3):575-582.

34. Klein S, Staring M, Murphy K, Viergever MA, Pluim JP. elastix: a toolbox for intensity-based medical image registration. IEEE Trans Med Imaging. 2010;29(1):196-205.

35. Holm S. A Simple Sequentially Rejective Multiple Test Procedure. Scand J Stat. 1979;6(2):65-70.

36. Weinreb JH, Sheth C, Apostolakos J, et al. Tendon structure, disease, and imaging. Muscles Ligaments Tendons J. 2014;4 (1):66-73.

37. Du J, Carl M, Diaz E, et al. Ultrashort TE T1rho (UTE T1rho) imaging of the Achilles tendon and meniscus. Magn Reson Med. 2010;64(3):834-842.

38. Du J, Bydder M, Takahashi AM, Carl M, Chung CB, Bydder GM. Short T2 contrast with three-dimensional ultrashort echo time imaging. Magnetic resonance imaging. 2011;29(4):470-482.

39. Juras V, Zbyn S, Pressl C, et al. Regional variations of $\mathrm{T}_{2}{ }^{*}$ in healthy and pathologic achilles tendon in vivo at 7 Tesla: preliminary results. Magn Reson Med. 2012;68(5):1607-1613.

40. Weiger M, Brunner DO, Dietrich BE, Müller CF, Pruessmann KP. ZTE imaging in humans. Magn Reson Med. 2013;70(2): 328-332.

41. Larson PE, Han M, Krug R, et al. Ultrashort echo time and zero echo time MRI at 7T. MAGMA. 2015.

42. Staroswiecki E, Bangerter NK, Gurney PT, Grafendorfer T, Gold GE, Hargreaves BA. In vivo sodium imaging of human patellar cartilage with a $3 \mathrm{D}$ cones sequence at $3 \mathrm{~T}$ and $7 \mathrm{~T}$. Journal of magnetic resonance imaging: JMRI. 2010;32(2): 446-451.

43. Lu A, Atkinson IC, Claiborne TC, Damen FC, Thulborn KR Quantitative sodium imaging with a flexible twisted projection pulse sequence. Magn Reson Med. 2010;63(6):15831593.

44. Grosse U, Syha R, Hein T, et al. Diagnostic value of T1 and T2 * relaxation times and off-resonance saturation effects in the evaluation of Achilles tendinopathy by MRI at 3T. Journal of magnetic resonance imaging: JMRI. 2015;41(4):964-973.

45. Karlsen OT, Verhagen R, Bovée WM. Parameter estimation from Rician-distributed data sets using a maximum likelihood estimator: application to $\mathrm{T} 1$ and perfusion measurements. Magn Reson Med. 1999;41(3):614-623.

46. Raya JG, Dietrich O, Horng A, Weber J, Reiser MF, Glaser C. T2 measurement in articular cartilage: impact of the fitting method on accuracy and precision at low SNR. Magnetic resonance in medicine: official journal of the Society of Magnetic Resonance in Medicine / Society of Magnetic Resonance in Medicine. 2010;63(1):181-193. 